PROGRESS REPORT

Grant DE-FG02-92 ER14289, First Period - 8/1/92 to 4/1/93

\title{
AN INVESTIGATION OF MOLYBDENUM AND MOLYBDENUM OXIDE CATALYZED HYDROCARBON FORMATION REACTIONS
}

\section{Overall Progress.}

Progress has been made during the first six months of this research project in the following areas:

1. Investigation of the Activity of Model Catalysts for Olefin Metathesis.

As outlined in the research proposal, the first step in the investigation is to identify the most active model oxide catalyst for propylene metathesis. Model catalysts were prepared by:

(i) Direct oxidation of metallic molybdenum. In this case, the full range of molybdenum oxidation states between 0 and +6 is accessible depending on the oxidation conditions.

(ii) Reduction of $\mathrm{MoO}_{3}$ in hydrogen using a protocol that mimics supported catalyst preparation techniques.

It was found that $\sim 0.5 \mathrm{ML}$ (monolayers) of oxygen (corresponding to a surface metal oxidation state of $\sim+1^{1}$ ) resulted in an enhancement in metathesis activity for the reaction:

$$
2 \mathrm{C}_{3} \mathrm{H}_{6} \rightarrow \mathrm{C}_{4} \mathrm{H}_{8}+\mathrm{C}_{2} \mathrm{H}_{4}
$$

but that higher oxygen coverages (and oxidation states) caused a significant diminution in activity (all compared to metallic molybdenum). This suggests that co-adsorption of an electron-

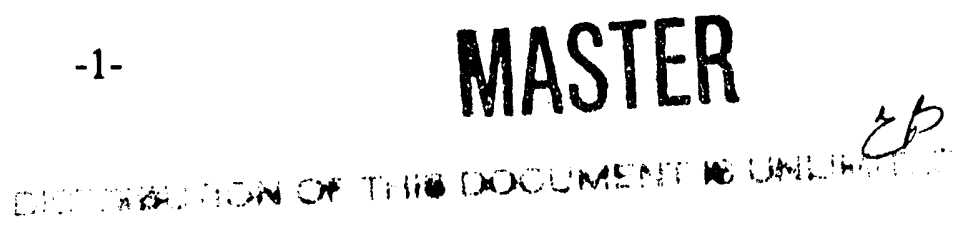


withdrawing additive enhances metathesis activity. The lower activity at higher oxygen coverages can be ascribed to the effects of site blocking.

Formation of $\mathrm{MoO}_{2}$ by reduction of $\mathrm{MoO}_{3}$ in hydrogen ${ }^{2}$ results in little enhancement in catalytic activity compared to metallic molybdenum. However, subsequent annealing of $\mathrm{MoO}_{2}$ (formed by $\mathrm{MoO}_{3}$ reduction) and, indeed, $\mathrm{MoO}_{3}$ itself, both in vacuo, leads to a significant enhancement in activity. This effect is suggested to be due to the thermal removal of surface oxygen to reveal the underlying molybdenum ions.

A similar annealing protocol for $\mathrm{MoO}_{2}$, in this case, formed directly by mild oxidation of metallic molybdenum ${ }^{3-9}$ results in a significant enhancement in metathesis activity. A histogram displaying the relative activity of the best metal oxides, all formed by direct oxidation and subsequent vacuum annealing is shown in Figure 1 where reaction turnover frequencies for $\mathrm{MoO}_{2}$ and $\mathrm{MoO}_{3}$ are compared with metallic molybdenum. Note that a significant enhancement is observed for $\mathrm{MoO}_{2}$ and a moderate enhancement for $\mathrm{MoO}_{3}$.

Reaction rates for all oxides, both with and without vacuum annealing displayed an induction period (i.e., reaction rates that depended on reaction time). The data displayed in 
Figure 1 are for the steady-state rate following completion of any induction period. Since the induction period is present for both vacuum-annealed (without an oxygen outer layer) and vacuum-unannealed (with the presence of an oxygen outer layer), this suggests that in situ catalyst reduction is not responsible for the presence of the induction period.

Finally, it was noted that the cis-2-butene to trans-2-butene ratio in the products from the metathesis of propylene exceeded the thermodynamic ratio for the reaction conditions.

The Activity of Molybdenum and Molybdenum Oxide Model Catalysts for Olefin Metathesis, B. Bartlett, C. Soto, R. Wu and W.T. Tysoe, Catalysis Letters, submitted

\section{The Kinetics of Mo(100)-catalyzed Olefin Metathesis.}

The kinetics of olefin metathesis catalyzed by a $\mathrm{Mo}(100)$ single crystal was measured as a function of propylene pressure and temperature. While metallic molybdenum is not conventionally thought of as a good metathesis catalyst, as shown in the previous section, it can catalyze metathesis but at a lower rate than molybdenum oxides. The reaction rate $r_{m}$ can be summarized as:

$$
r_{m}=8 \pm 2 \times 10^{12} p\left(C_{3} H_{6}\right)^{1.0 \pm 0.1} \exp \left(-65 \pm 5 \times 10^{3} / R T\right)
$$

where $r_{m}$ is given in reactions/site/s where a site is taken to be a square unit cell on a clean $\mathrm{Mo}(100)$ surface, $\mathrm{p}$ is the propylene pressure in Torr and $\mathrm{T}$ the reaction temperature. Reaction rates measured on a foil agree well with values calculated using equation 1 suggesting that the reaction is not strongly structure-sensitive. It is also found that reaction proceeds in the presence of a thick ( $\sim 10 \mathrm{ML})$ carbonaceous layer. However, the large value of activation energy for this 
reaction: suggests that a highly activated rate-limiting step is responsible for the low overall rate.

The Kinetics of Propylene : Yetathesis Catalyzed by a Mo(100) Single Crystal. L.P. Wang, C. Soto and W.T. Tysoe, Journal of Catalysis, submitted

\section{Surface Chemistry of Tricyclic Molecules: Ethylene Oxide on Pd(111).}

We proposed to attempt to form surface metallocycles by cyclopropane adsorption on model catalysts. In order to further explore the feasibility of this experimental approach, we have investigated the surface chemistry of ethylene oxide $\left(\mathrm{C}_{2} \mathrm{H}_{2} \mathrm{O}\right)$ on pailadium. The experiments were also carried out to test the hypothesis that the surface reaction route for ethylene epoxidation parallels that for olefin metathesis as shown in scheme 1 , where $\mathrm{X}$ is $\mathrm{CH}_{2}$ for metathesis and $\mathrm{O}$ for reaction with oxygen.

$$
\rightarrow \quad \mathrm{CH}_{3}-\mathrm{CH}=\mathrm{X}
$$

$$
\begin{aligned}
& \underline{\mathrm{X}}+\mathrm{C}_{2} \mathrm{H}_{4} \stackrel{\mathrm{CH}_{2}}{/ \mathrm{CH}_{\mathrm{X}}^{\mathrm{CH}}} \rightarrow \mathrm{CH}_{2}=\mathrm{X}+\mathrm{CH}_{2-} \\
& \rightarrow \quad \mathrm{CH}_{\mathrm{X}}-\mathrm{CH}_{2}
\end{aligned}
$$

\section{Scheme I}

Here, path (a) corresponds to a $\beta$-hydrogen transfer reaction of the metallocycle, (b) gives rise to metathesis when $\mathrm{X}$ is $\mathrm{CH}_{2}$ and (c) yields ethylene oxide when $\mathrm{X}$ is $\mathrm{O}$. The chemistry of ethylene oxide on $\mathrm{Pd}(111)$ was studied using temperature programmed desorption (TPD). 
Palladium was selected since it is slightly more reactive than silver, the catalyst for ethylene epoxidation, and the chemistry of the reaction products are well understood on this surface. ${ }^{10-12}$ The observed desorption products and temperatures were consistent with scheme I. This result indicates the general feasibility of the proposed approach to forming surface metallocycles and also indicates the validity of the proposed Scheme I.

The Thermal Decomposition of Ethylene Oxide on Pd(111): Comparison of the Reaction Pathways for the Selective Oxidation of Ethylene and Olefin Metathesis. W.T. Tysoe, R.M. Ormerod and R.M. Lambert, Journal of the American Chemical Society, submitted

\section{UV Source Construction.}

As indicated in the proposal, an ultraviolet source based on a design by Rabelais ${ }^{13}$ is being constructed. This has been completed and preliminary tests indicate that the source works well. Some recent modifications have been made, in particular in the differential pumping, to improve the chamber pressure during operation. The source requires further testing and characterization but preliminary results appear to be promising.

References.

1. J.L. Grant, T.B. Fryberger and P.C. Stair, Surf. Sci., 159, 333 (1985)

2. P.A. Spevack and N.S. McIntyre, J. Phys. Chem., 96, 9029 (1992)

3. H.M. Kennett and A.E. Lee, Surf. Sci. 48, 591 (1975)

4. H.M. Kennett and A.E. Lee, Surf. Sci. 48, 606 (1975)

5. H.M. Kennett and A.E. Lee, Surf. Sci. 48, 617 (1975) 
6 : H.M. Kennett and A.E. Lee, Surf. Sci. 48, 624 (1975)

7. H.M. Kennett and A.E. Lee, Surf. Sci. 48, 633 (1975)

8. A.E. Lee, Surf. Sci. 47, 191 (1975)

9. C. Zhang, M.A. Van Hove and G.A. Somorjai, Surf. Sci. 149, 326 (1985)

10. W.T Tysoe, G.L. Nyberg and R.M. Lambert, J. Phys. Chem. 88, 19601984

11. L.P. Wang, W.T. Tysoe, R.M. Ormerod, R.M. Lambert, H. Hoffmann, and F. Zaera, J. Phys. Chem. 94, 4236 (1990)

12. J.L Davis and M.A. Barteau, J. Am. Chem. Soc. 111, 1782 (1989)

13. G.M. Lancaster, J.A. Taylor, A. Ignatiev and J.W. Rabelais, J. Elec. Spec. and Rel. Phenom., 14, 143 (1978)

II. List of Publications.

1. The Activity of Molybdenum and Molybdenum Oxide Model Catalysts for Olefin Metathesis, B. Bartlett, C. Soto, R. Wu and W.T. Tysoe, Catalysis Letters, submitted

2. The Kinetics of Propylene Metathesis Catalyzed by a Mo(100) Single Crystal. L.P. Wang, C. Soto and W.T. Tysoe, J. Catal, submitted

3. The Thermal Decomposition of Ethylene Oxide on Pd(111): Comparison of the Reaction Pathways for the Selective Oxidation of Ethylene and Olefin Metathesis. W.T. Tysoe, R.M. Ormerod and R.M. Lambert, J. Am. Chem. Soc., submitted

\section{Plan for Upcoming Year}

The work for the immediate future is broadly divided into two aspects:

(i) Surface chemistry and characterization of the model oxide catalysts.

(ii) Kinetic studies of the model oxide catalysts. 
$\therefore \quad$ There will be a close interplay between these two aspects and insights gained in one area will be used to guide investigation in another.

\section{Surface Chemistry and Characterization of Model Oxide Catalysts}

We will characterize the active model $\mathrm{MoO}_{2}$ and $\mathrm{MoO}_{3}$ catalysts (Figure 1) so that we can reproducibly make oxygen-terminated and molybdenum-terminated catalysts. These oxides will be characterized using temperature programmed desorption, Auger spectroscopy, electron energy loss spectroscopy, $\mathrm{X}$-ray and ultraviolet photoelectron spectroscopies and work-function measurements.

We will initially probe the chemistry of these surface using simple reactants, $\mathrm{CO}$ or NO, first to titrate surface sites and second to compare the reactivity of these sites with metallic molybdenum where the chemistry of $\mathrm{CO}$ and $\mathrm{NO}$ is well understood.

We will also examine the chemistry of small hydrocarbons on the metal oxide surfaces.

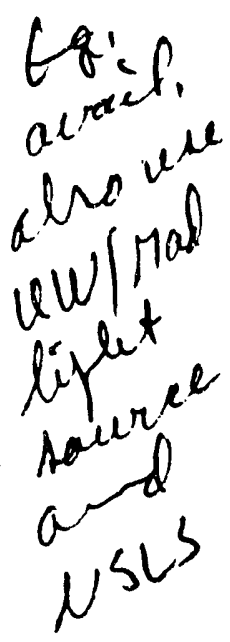
Preliminary results in this area indicate the appearance of apparentiy very large carbon signals following, for example, propylene adsorption on $\mathrm{MoO}_{2}$ as measured using Auger spectroscopy. These intriguing results require further confirmation but do indicate the feasibility of studying the surface chemistry of hydrocarbons on the oxide surfaces. Of particular interest is to study cyclopropane adsorption in experiments analogous to those described in section 1.3 for ethylene oxide on $\operatorname{Pd}(111)$. A similar surface chemistry for cyclopropane should result in metallocycle formation and subsequent thermal decomposition yielding ethylene. The activation energy for this step (measured using TPD) compared with the reaction activation energy will provide insights into the kinetics of some of the elementary steps in the "carbene" mechanism. 


\section{Kingtic Studies of Model Oxide Catalysts}

Kinetic studies will focus on the "active" catalysts, namely $\mathrm{MoO}_{2}, \mathrm{MoO}_{3}$ and $0.5 \mathrm{ML}$ O/Mo. These catalysts display induction periods and preliminary results suggest that reduction of the surface is not responsible for this effect. The temperature and pressure dependencies of the induction period and the steady-state rate will be measured. A possible explanation for the existence of an induction period, if surface reaction does proceed via a carbene mechanism, is that the induction period just reflects the carbene formation kinetics. If this is true, prior formation of carbene by cyclopropane decomposition should eliminate, or at least mitigate, the induction period. The presence or absence of the induction period following restart reactions should also provide insights into the nature of the induction period.

Finally, we have noted that the cis-2-butene to trans-2-butene ratio in the metathesis products significantly exceeds that expected thermodynamically. This effect will be investigated further. 

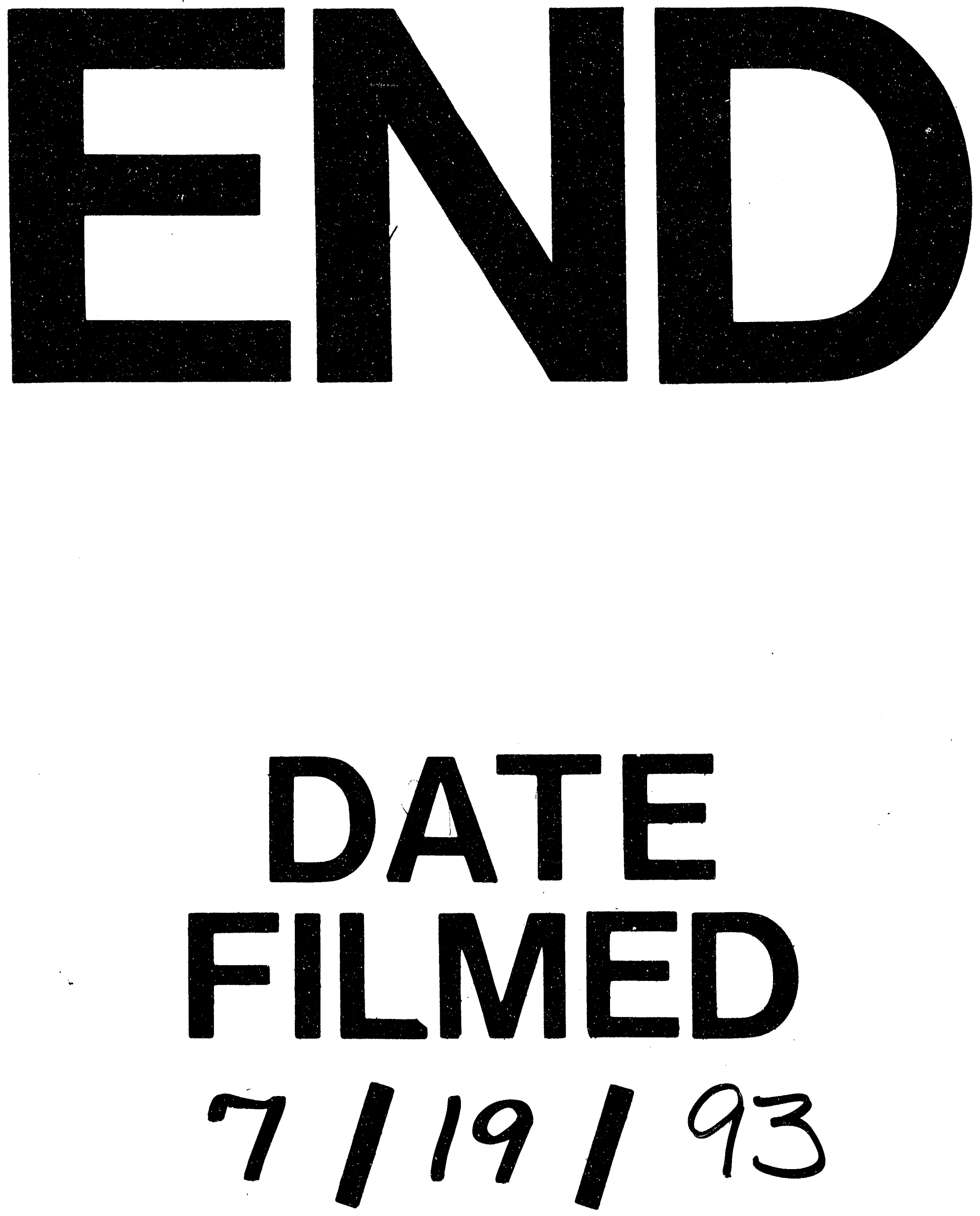
